\title{
Effects of early nutrition on the infant metabolome
}

Christian Hellmuth ${ }^{1}$, Olaf Uhl ${ }^{1}$, Franca F. Kirchberg ${ }^{1}$, Veit Grote ${ }^{1}$, Martina Weber ${ }^{1}$, Peter Rzehak $^{1}$, Clotilde Carlier ${ }^{2}$, Natalia Ferre ${ }^{3}$, Elvira Verduci ${ }^{4}$, Dariusz Gruszfeld ${ }^{5}$, Piotr Socha ${ }^{5}$, Berthold Koletzko ${ }^{1}$; European Childhood Obesity Trial Study Group

${ }^{1}$ Dr. von Hauner Children's Hospital, Ludwig-Maximilians-University of Munich, München, Germany

${ }^{2}$ University Children's Hospital Queen Fabiola, Université Libre de Bruxelles, Brussels, Belgium

${ }^{3}$ Paediatrics Research Unit, Universitat Rovira i Virgili, Reus, Spain

${ }^{4}$ San Paolo Hospital, University of Milan, Milano, Italy

${ }^{5}$ Children's Memorial Health Institute, Warsaw, Poland

Short Title: Early nutrition and the infant metabolome

Correspondence to

Dr Christian Hellmuth

Div. Metabolic and Nutritional Medicine

Dr. von Hauner Children's Hospital, Ludwig-Maximilians-University of Munich

Lindwurmstr. 4, D-80337 Muenchen, Germany

Phone: +49 (0)8944005 7991, Fax: +49 (0)89 440057742

Email: Christian.hellmuth@med.uni-muenchen.de 


\begin{abstract}
Breastfeeding induces a different metabolic and endocrine response than feeding conventional infant formula, and it has also been associated with slower weight gain and reduced disease risk in later life. The underlying programming mechanisms remain to be explored. Breastfeeding has been reported to induce lower levels of insulin, IGF-1 and some amino acids than formula feeding. In the CHOP trial, infants fed conventional protein-rich formula had a higher BMI at 2 years and 6 years than those fed a protein reduced formula. At 6 month, higher protein intakes induced increased plasma concentrations of branched-chain amino acids (BCAA) and their oxidation products, short-chain acylcarnitines. With increasing BCAA, these short-chain acylcarnitines increased proportional only until a break point was reached, after which BCAA seemed to escape their degradation. The resulting marked elevation of BCAA with high protein intakes appears to contribute to increased insulin levels and to affect $\beta$-oxidation of fatty acids. The ratios of long-chain acylcarnitines to free carnitine decreased in infants who received high protein formula, which indicates a reduced initiation of B-oxidation. We conclude that high protein intakes inducing high BCAA plasma levels may inhibit fat oxidation and thereby enhance body fat deposition and adiposity.
\end{abstract}

Key Words: BCAA/ Acylcarnitines / Programming/ High-protein / Metabolomics 
The infant's metabolic response adapts to environmental and particularly dietary exposure and appears to affect growth, body composition, and later disease risks [1]. Compared to feeding conventional infant formula, breastfeeding was shown to induce a different metabolic and endocrine response, and it has been associated with differences in growth, body composition and disease risk throughout childhood and in adult life [2]. Populations of breastfed (BF) children show a lower prevalence of overweight and obesity at school age. The "Early Protein Hypothesis“ links the amount of protein in infant feeding to weight gain in the first months of life, which is related to obesity risk in childhood and early adulthood [3]. The underlying programming mechanisms remain to be explored. Recent publications point to epigenetic modifications of genes affecting different proteins and hormones, like leptin, insulin-like growth factor I (IGF-I) and insulin [4]. Associations of endocrine and hormonal markers with obesity are discussed by Socha et al. (Socha et al., NNW series 2015, this volume).

\section{Effect of infant formula on the metabolome}

Regardless the primary mechanism, changes will be induced in the metabolome of infants fed different diets, since metabolites (molecules <1500 Da) are the downstream products of both genetic and epigenetic alterations as well as environmental factors, including diet. The metabolome is closely related to the phenotype. More important, metabolomics is capable to enhance the understanding of metabolic regulation in response to environmental influences [5]. It is expected that the "programming effects" of infant formula with different contents of protein should be reflected in the metabolome and consequently in the regulation of metabolic pathways. Martin and coworkers found differences in the metabolism of formula fed (FF) babies from obese mothers compared to BF infants [6]. Higher short-chain acylcarnitines $C 2$, C3, C4 and amino acids (AA) were found in stool of FF infants suggesting an increased breakdown of protein in the gut by bacteria. Differences in the urinary metabolome pointed to an increased protein metabolism in the FF infants. Furthermore, beta-oxidation and ketogenesis were affected by formula feeding. In 1998, Karlsland Akeson et al. reported increased values of some essential amino acids in blood plasma of healthy infants at age of 6 month, who were exclusively breast-fed until the age of 3 months and afterwards were randomly assigned to infant formulae with either 13,15 , or $18 \mathrm{~g} / \mathrm{l}$ protein (Table 1) [7]. Since the sample number was very small and the mothers were allowed to breast-feed as long as they wished and to introduce the assigned formulas gradually, the effect of the formula intake was vanished. In the BeMIM study, infants of mothers who chose to formula feed received 
either a standard formula $(2.2 \mathrm{~g} / 100 \mathrm{kcal}$ protein) or an intervention formula with lower protein content $(1.89 \mathrm{~g} / 100 \mathrm{kcal}$ protein $)$ and modified protein composition, which was introduced within 28 days after birth [8]. The intervention formula contained additional alphalactalbumin, free phenylalanine, free tryptophan and long-chain polyunsaturated fatty acids. A breastfed group was also followed for reference. Urea and AA, in particular non- dispensable AA, were higher in the blood plasma of both groups of FF infants (Table 1). Non-essential or dispensable AA were less affected by the different diets or even decrease in the plasma of FF infants, e.g. glutamine, glutamate or serine.

\section{CHOP - lower vs. higher protein intake}

In a large, double blind randomized clinical intervention trial, we studied the effect of infant and follow-on formula with conventionally higher (HP, $2.05 \mathrm{~g} / 100 \mathrm{ml}$ protein) or reduced protein contents (LP, $1.25 \mathrm{~g} / 100 \mathrm{ml}$ protein) on infant growth and metabolic response. In this trial, the higher protein formula led to higher BMI than lower protein formula at 2 years of age [9] and at school age [10]. Total IGF-I serum levels were increased in the HP group, whereas IGF-binding protein 2 (IGF-BP2) was lower and IGF-BP3 did not differ significantly between both formula groups at the age of 6 months [11].

At the age of 6 months, HP fed children showed significantly increased plasma concentrations of non-dispensable amino acids (Table1), including the branched-chain amino acids (BCAA; Ile, Leu, Val) [11] as well as increased levels of the oxidation products of BCAA, short-chain acylcarnitines, compared to LP intake as well as to a reference group of BF infants (Table 2) [12]. Also, urea increased significantly in both the LP and HP groups compared to the BF.

\section{BCAA metabolism in formula fed infants}

Given that elevated levels of BCAA and their degradation products are associated with infant formula intake, the BCAA metabolism might be the potential key factor in the relation between formula feeding and later obesity development. BCAA are less metabolized during the first pass in the liver compared to other AA [13]. In general, dietary proteins are degraded in the intestine to peptides and free AA, which are resorbed. After intestinal resorption and metabolism, the portal vein transports the AA to the liver where they undergo first pass metabolism. However, the key enzyme of BCAA oxidation, branched-chain $\alpha$-keto acid 
dehydrogenase $(\mathrm{BCKDH})$, is less present in the liver [13]. Thus, the BCAA output of the liver is enhanced compared to other AA and BCAA are much more increased in the plasma of HPfed children than other essential amino acids.

In the skeletal muscle, BCAA are degraded for energy provision [14]. First, valine, leucine, and isoleucine are transaminased by the branched-chain amino transferase (BCAT) to $\alpha$-ketoacids (Figure 1). These keto-acids are subsequently reduced by BCKDH to short-chain fatty acids) which are bound to carnitine to short-chain acylcarnitines C4 and C5 [15]. Further degradation products comprise the acylcarnitine $\mathrm{C} 3, \mathrm{C} 5 . \mathrm{OH}$ and $\mathrm{C} 5.1$. The reduction step via $\mathrm{BCKDH}$ is the limiting factor in the degradation of BCAA [13]. Leucine supplementation increases BCKDH activity [14] to ensure higher degradation of BCAA in status of high BCAA availability to keep BCAA levels in a physiological range. In infants in the CHOP trial, we demonstrated a saturation of this pathway. Segmented regression models revealed that with increasing BCAA, the short-chain acylcarnitines only increased until a break point was reached (Figure 2) [12]. After this point, the corresponding short-chain acylcarnitines $C 4$ and C5 did not longer increase with increasing BCAA concentration. Thus, BCAA seemed to escape their degradation after a certain point of high plasma BCAA levels, which indicates a saturation of BCAA catabolism in infants. This was especially observed for the HP group, who reached higher plasma levels of BCAA. To our knowledge, this is the first indication, that above a certain plasma concentration, the BCAA degradation pathway becomes saturated. This could potentially be of major biological importance in infants fed high amount of protein, and where a markedly increased risk of adverse effects mediated through BCAA may result. Leucine, for instance, is a potent stimulator of insulin secretion [16]. Increased cpeptide/creatinine ratios in HP fed infants were shown in the CHOP trial [6].

Furthermore, leucine depressed beta-oxidation of fatty acids [17]. The ratios of long-chain acylcarnitines to free carnitine decreased in infants who received HP formula in the CHOP trial, which indicates a lower initial step of the B-oxidation (Table 3) [12]. Moreover, leucine deprivation resulted in reduced activity of fatty synthase genes [18]. This deregulation on fat metabolism may result in a lipid oversupply, which causes consequently lipotoxicity, insulin resistance, and fat storage [19]. Thus, high protein and BCAA intake may inhibit fat oxidation and thereby enhance body fat deposition and the risk of adiposity. This would explain the effects of HP feeding on increased weight gain during the first years of live. The absence of significant differences for leucine and isoleucine as well as acylcarnitines C4 and C5 between LP-fed and BF infants underline the influence of HP-feeding on the metabolism. 


\section{Other indispensable AA}

Infant protein supply also affects the metabolism of other AA. Aromatic amino acids (AAA) particularly phenylalanine, which promotes IGF-1 secretion, were also elevated in the plasma of infants fed conventional FF infants (Table 1). Levels of the non-essential AAA tyrosine are also increased in the HP group due to transformation of phenylalanine to tyrosine [20]. The trials showed that HP diet resulted in higher levels of AA compared to LP diet (Table 1). BCAA and AAA compete for transportation in mammalian tissues [21]. Therefore, higher values of BCAA result in a lower uptake of AAA, e.g. in the brain, and higher AAA plasma levels [22]. Reduction of AAA levels in the brain lower the synthesis and the release of neurotransmitters like serotonin and catecholamines. This affects metabolic pathways. As BCAA, AAA are insulinogenic [16] and elevated levels are related to obesity and may predict future diabetes [23]. Regarding the effect of AAA on IGF-1 levels, AAA may represent the missing link between HP intake or leucine supplementation and elevated IGF-1 levels [24]. In conclusion, not only the amount of protein in formula, but also the composition and the kind of the protein used are related the later adverse outcomes of FF infants.

Nearly all other essential amino acids were elevated in FF infants and were particularly strongly affected by HP diet (Table 1). Higher concentrations of certain essential amino acids, namely leucine, phenylalanine, tyrosine, and lysine, are well known to contribute to an elevated insulin secretion [16]. In contrast, a contribution of elevated essential AA to growth hormone and IGF-1 levels may be assumed, but further investigations in human intervention studies are needed to get a more detailed picture of the underlying molecular mechanisms. However, elevated essential AA and their effect on the secretion of growth factors may be an underlying mechanism of the Early Protein Hypothesis [3].

\section{Dispensable amino acids - the decrease of glutamine}

Nonessential AA are less affected by HP diet than indispensable AA. The lesser influence of the diet on non-essential AA appears to be due to regulating mechanisms. Since these AA are endogenously synthesized, the human metabolism can down-regulate the biosynthesis of these AA in times of protein over-supply to keep levels in the tissues and the blood plasma constant. This regulation mainly appears in the intestine and the liver during the first pass, 
hence, in contrast to oral supplements, direct infusion may affect plasma levels. Not in conclusion with this hypothesis, glutamine levels are decreased in different studies investigating FF infants (Table 1). In the CHOP trial, glutamine was lower in the HP group compared to LP-fed infants and even the LP-fed infants had lower levels compared to BF infants. An alteration in the urea cycle is assumed, because urea is elevated in FF infants $[8,11,25]$. However, levels of other AA involved in the urea cycle, namely glutamic acid, aspartic acid, arginine, and ornithine, showed no consistent picture or were not affected by formula diet (Table 1), whereas citrulline was elevated in the CHOP and in the BeMIM trial. In contrast to glutamine, cells can recycle the other amino acids during the urea cycle or aspartate cycle [26]. Glutamic acid can be recycled at the expense of glutamine. Thus, glutamine may be the only AA which levels are decreased by an elevated urea cycle. Elevation of the urea cycle in the formula groups could result from enhanced protein intake and the subsequent higher protein metabolism. Another explanation of the lower glutamine levels in the HP group might be the contribution of glutamine for insulin secretion induced by leucine [27]. Leucine activates the glutamate dehydrogenase in pancreatic islets resulting in consumption of glutamic acid. In pancreatic islets, glutamic acid is mainly provided by the intra-cellular conversion of glutamine to glutamic acid [28]. Hence, increased insulin release, enhanced by leucine levels, may decrease glutamine levels.

\section{One step further - the link to early weight gain and obesity risk}

Metabolites that responded to a high protein infant supply have been previously reported as markers for obesity risk. BCAA, non-esterified fatty acids, organic acids, acylcarnitines and phospholipids were identified as potential biomarkers for obesity in a recent review [29]. This indicates a relation of elevated BCAA by HP diet to the obese state [21,23]. Furthermore, a deregulation of the beta-oxidation seems to be associated with development of obesity and insulin resistance. Nevertheless, the underlying mechanisms and pathways require further exploration. The CHOP trial offers the possibility to analyze the onset of obesity and the change of metabolites over the period of obesity development longitudinally. For instance, it was shown in the CHOP trial that lysophosphatidylcholine 14:0 is strongly related to rapid weight gain in infancy in the first 6 month of life and to overweight/obesity at the age of 6 years [30]. However, unraveling the effects of infant formula on the metabolome remains challenging and further trials will provide insights in the molecular mechanism and help to optimize infant formula. Metabolites like keto-acids or intermediates of the citric acid cycle or 
from gluconeogenesis should be analyzed in response the formula feeding and may give new insights in the future.

\section{Conclusion}

A high protein intake in excess of metabolic requirements increases BCAA concentrations in infant plasma to levels at which the normal catabolic capacity for BCAA is exceeded.

Thereby, high dietary protein supply to infants may stimulate markedly enhanced secretion of the growth factors insulin and IGF-1 and induce signaling effects inducing excessive weight gain. Moreover, a high protein intake in infants appears to inhibit initiation of beta-oxidation and thus may contribute to enhanced fat storage and increased adiposity, probably by enhanced BCAA levels. Elevated levels of BCAA and disturbed beta-oxidation have been shown in previous observational studies to be associated with obesity and cardiovascular risk. Thus, BCAA metabolism might present a mechanism linking infant formula feeding and obesity risk. 


\section{Acknowledgement}

This work was financially supported by the Commission of the European Communities, the seventh Framework Programme, contract FP7-289 346-EARLY NUTRITION, and the European Research Council Advanced Grant ERC-2012-AdG - no.322605 META-

GROWTH. This manuscript does not necessarily reflect the views of the Commission and in no way anticipates the future policy in this area. The funders had no role in study design, data collection and analysis, decision to publish, or preparation of the manuscript. Funds to support the writing of the manuscript were provided by Nestlé Nutrition, Vevey, Switzerland. 


\section{Appendix}

The European Childhood Obesity Project Study Group:

Philippe Goyens, Clotilde Carlier, Joana Hoyos (ULB, Bruxelles, Belgium), Françoise Martin, Annick Xhonneux, Jean-Paul Langhendries, Jean-Noel Van Hees (CHC StVincent, Liège, Belgium), Ricardo Closa-Monasterolo, Joaquin Escribano, Veronica Luque, Georgina, Mendez, Natalia Ferre, Marta Zaragoza-Jordana (Universitat Rovira I Virgili, Tarragon, Spain), Marcello Giovannini, Enrica Riva, Carlo Agostoni, Silvia Scaglioni, Elvira Verduci, Fiammetta Vecchi, Alice Re Dionigi (University of Milan, Italy), Jerzy Socha, Anna Dobrzańska, Dariusz Gruszfeld, Piotr Socha, Anna Stolarczyk, Agnieszka Kowalik, Roman Janas, Ewa Pietraszek (Children's Memorial Health Institute, Warsaw, Poland), Emmanuel Perrin (Danone Research Centre for Specialized Nutrition, Schiphol, The Netherlands), Berthold Koletzko, Veit Grote, Martina Weber, Sonia Schiess, Jeannette Beyer, Michaela Fritsch, Uschi Handel, Ingrid Pawellek, Sabine Verwied-Jorky, Iris Hannibal, Hans Demmelmair, Gudrun Haile, Wolfgang Peissner, Ulrike Harder, Franca Kirchberg, Olaf Uhl (Dr. von Hauner Childrens Hospital, University of Munich, Germany), Rüdiger von Kries (Institute for Social Paediatrics and Adolescent Medicine, University of Munich, Germany). 


\section{References $(\max 30)$}

1 Koletzko B, Brands B, Chourdakis $\mathrm{M}$, et al.: The power of programming and the earlynutrition project: Opportunities for health promotion by nutrition during the first thousand days of life and beyond. Ann Nutr Metab 2014;64:187-196.

Arenz S, Ruckerl R, Koletzko B, et al.: Breast-feeding and childhood obesity--a systematic review. Int J Obes Relat Metab Disord 2004;28:1247-1256.

Koletzko B, von Kries R, Closa R, et al.: Can infant feeding choices modulate later obesity risk? Am J Clin Nutr 2009;89:1502S-1508S.

Ruchat S-M, Bouchard L, Hivert M-F: Early infant nutrition and metabolic programming: What are the potential molecular mechanisms? Curr Nutr Rep 2014;3:281-288. Nicholson JK, Lindon JC: Systems biology: Metabonomics. Nature 2008;455:1054-1056.

6 Martin FP, Moco S, Montoliu I, et al.: Impact of breast-feeding and high- and low-protein formula on the metabolism and growth of infants from overweight and obese mothers. Pediatr Res 2014;75:535-543.

7 Karlsland Akeson PM, Axelsson IE, Raiha NC: Protein and amino acid metabolism in three- to twelve-month-old infants fed human milk or formulas with varying protein concentrations. J Pediatr Gastroenterol Nutr 1998;26:297-304.

8 Fleddermann M, Demmelmair $\mathrm{H}$, Grote V, et al.: Infant formula composition affects energetic efficiency for growth: The bemim study, a randomized controlled trial. Clin Nutr 2014;33:588595.

$9 \quad$ Koletzko B, von Kries $\mathrm{R}$, Closa $\mathrm{R}$, et al.: Lower protein in infant formula is associated with lower weight up to age 2 y: A randomized clinical trial. Am J Clin Nutr 2009;89:1836-1845. Weber M, Grote V, Closa-Monasterolo R, et al.: Lower protein content in infant formula reduces bmi and obesity risk at school age: Follow-up of a randomized trial. Am J Clin Nutr 2014;99:1041-1051.

11 Socha P, Grote V, Gruszfeld D, et al.: Milk protein intake, the metabolic-endocrine response, and growth in infancy: Data from a randomized clinical trial. Am J Clin Nutr 2011;94:1776S1784 S.

12 Kirchberg FF, Harder $\mathrm{U}$, Weber $\mathrm{M}$, et al.: Dietary protein intake affects amino acid and acylcarnitine metabolism in infants aged 6 months. J Clin Endocrinol Metab 2014:jc20143157.

13 Brosnan JT, Brosnan ME: Branched-chain amino acids: Enzyme and substrate regulation. J Nutr 2006;136:207S-211S.

14 Shimomura Y, Murakami T, Nakai N, et al.: Exercise promotes bcaa catabolism: Effects of bcaa supplementation on skeletal muscle during exercise. J Nutr 2004;134:1583S-1587S.

15 Roe DS, Roe CR, Brivet M, et al.: Evidence for a short-chain carnitine-acylcarnitine translocase in mitochondria specifically related to the metabolism of branched-chain amino acids. Mol Genet Metab 2000;69:69-75.

16 Kuhara T, Ikeda S, Ohneda A, et al.: Effects of intravenous infusion of 17 amino acids on the secretion of gh, glucagon, and insulin in sheep. Am J Physiol 1991;260:E21-26.

17 Goichon A, Chan P, Lecleire S, et al.: An enteral leucine supply modulates human duodenal mucosal proteome and decreases the expression of enzymes involved in fatty acid betaoxidation. J Proteomics 2013;78:535-544.

18 Cheng $\mathrm{Y}$, Meng $\mathrm{Q}$, Wang $\mathrm{C}$, et al.: Leucine deprivation decreases fat mass by stimulation of lipolysis in white adipose tissue and upregulation of uncoupling protein 1 (ucp1) in brown adipose tissue. Diabetes 2010;59:17-25.

19 Samuel VT, Petersen KF, Shulman GI: Lipid-induced insulin resistance: Unravelling the mechanism. Lancet 2010;375:2267-2277.

20 Matthews DE: An overview of phenylalanine and tyrosine kinetics in humans. J Nutr 2007;137:1549S-1555S; discussion 1573S-1575S. 
21 Newgard CB, An J, Bain JR, et al.: A branched-chain amino acid-related metabolic signature that differentiates obese and lean humans and contributes to insulin resistance. Cell Metab 2009;9:311-326.

22 Fernstrom JD: Branched-chain amino acids and brain function. J Nutr 2005;135:1539S-1546S.

23 Adams SH: Emerging perspectives on essential amino acid metabolism in obesity and the insulin-resistant state. Adv Nutr 2011;2:445-456.

24 Dawson-Hughes B, Harris SS, Rasmussen HM, et al.: Comparative effects of oral aromatic and branched-chain amino acids on urine calcium excretion in humans. Osteoporos Int 2007;18:955-961.

25 Picone TA, Benson JD, Moro G, et al.: Growth, serum biochemistries, and amino acids of term infants fed formulas with amino acid and protein concentrations similar to human milk. J Pediatr Gastroenterol Nutr 1989;9:351-360.

26 Haberle J, Boddaert N, Burlina A, et al.: Suggested guidelines for the diagnosis and management of urea cycle disorders. Orphanet J Rare Dis 2012;7:32.

27 Sener A, Malaisse WJ: L-leucine and a nonmetabolized analogue activate pancreatic islet glutamate dehydrogenase. Nature 1980;288:187-189.

28 Malaisse WJ, Sener A, Carpinelli AR, et al.: The stimulus-secretion coupling of glucoseinduced insulin release xlvi. Physiological role of I-glutamine as a fuel for pancreatic islets. Molecular and Cellular Endocrinology 1980;20:171-189.

29 Rauschert S, Uhl O, Koletzko B, et al.: Metabolomic biomarkers for obesity in humans: A short review. Ann Nutr Metab 2014;64:314-324.

30 Rzehak P, Hellmuth C, Uhl O, et al.: Rapid growth and childhood obesity are strongly associated with lysopc(14:0). Ann Nutr Metab 2014;64:294-303. 


\section{Tables}

Table 1 Plasma concentrations ( $\mu \mathrm{mol} / \mathrm{L})$ of amino acids in infants, who were formula fed or breastfed (BF) in three different studies.

\begin{tabular}{|c|c|c|c|c|c|c|c|c|c|}
\hline \multirow{3}{*}{$\begin{array}{l}\text { Fomula } \\
\text { Protein } \\
(\mathrm{g} / 100 \mathrm{kcal})\end{array}$} & \multicolumn{3}{|c|}{$\begin{array}{c}\text { CHOP } \\
6 \text { month }[11,12]\end{array}$} & \multicolumn{3}{|c|}{$\begin{array}{c}\text { BeMIM } \\
\text { 4month [8] }\end{array}$} & \multicolumn{3}{|c|}{$\begin{array}{c}\text { Karlsland Akeson et al. } \\
6 \text { month [7] }\end{array}$} \\
\hline & LP & HP & $\mathrm{BF}$ & LP & $\mathrm{HP}$ & $\mathrm{BF}$ & F19 & F22 & $\mathrm{F} 27$ \\
\hline & 1.8 & 2.9 & - & 1.9 & 2.2 & - & 1.9 & 2.2 & 2.7 \\
\hline Sample number & $\mathrm{n}=260$ & $\mathrm{n}=262$ & $\mathrm{n}=158$ & $\mathrm{n}=82$ & $\mathrm{n}=82$ & $\mathrm{n}=92$ & $\mathrm{n}=10$ & $\mathrm{n}=7$ & $\mathrm{n}=8$ \\
\hline Isoleucine & 64 & $85^{*}$ & 58 & $69^{*}$ & $80^{*}$ & 61 & 57 & $70 *$ & $69^{*}$ \\
\hline Leucine & 120 & $165^{*}$ & 106 & $127^{*}$ & $143^{*}$ & 121 & 99 & 120 & 117 \\
\hline Lysine & 166 & $197 *$ & 145 & $194 *$ & $190 *$ & 175 & 149 & 179 & 164 \\
\hline Methionine & 31 & $35^{*}$ & 24 & $31^{*}$ & $35^{*}$ & 25 & $25^{*}$ & $30^{*}$ & $33^{*}$ \\
\hline Phenylalanine & $72 *$ & $84 *$ & 61 & $65^{*}$ & $60 *$ & 48 & $53^{*}$ & $65^{*}$ & $60 *$ \\
\hline Threonine & 126 & $142 *$ & 119 & 140 & $184 *$ & 142 & 108 & $127 *$ & $135^{*}$ \\
\hline Tryptophan & 56 & 67 & 60 & $71 *$ & 66 & 65 & 49 & 57 & 60 \\
\hline Valine & $214^{*}$ & $304 *$ & 172 & $204 *$ & $232 *$ & 173 & 188 & $224 *$ & $225^{*}$ \\
\hline Alanine & 440 & 420 & 430 & 361 & 382 & 362 & 332 & 350 & 333 \\
\hline Arginine & 115 & 110 & 113 & 82 & 92 & 83 & 68 & 75 & 74 \\
\hline Asparagine & 54 & 58 & 52 & $50 *$ & $56^{*}$ & 44 & 44 & 50 & 55 \\
\hline Aspartate & 25 & 27 & 26 & 12 & $13 *$ & 11 & 10 & 12 & 12 \\
\hline Glutamine & $605^{*}$ & $556^{*}$ & 664 & $561 *$ & $559 *$ & 620 & $594 *$ & $582 *$ & 603 \\
\hline Glutamate & 122 & 115 & 130 & $142 *$ & $139 *$ & 171 & 89 & 74 & 76 \\
\hline Glycine & $267 *$ & 230 & 220 & 178 & 177 & 170 & 199 & 243 & 213 \\
\hline Histidine & $105^{*}$ & $107 *$ & 88 & 85 & 88 & 90 & 88 & 91 & 95 \\
\hline Serine & $161^{*}$ & $159 *$ & 187 & $135 *$ & $133^{*}$ & 142 & 149 & 149 & 152 \\
\hline Tyrosine & $83 *$ & $101 *$ & 66 & $92 *$ & 85 & 80 & $53^{*}$ & $65^{*}$ & $60 *$ \\
\hline Citrulline & $32 *$ & $34 *$ & 27 & $21^{*}$ & $22 *$ & 15 & 22 & 26 & 28 \\
\hline Ornithine & 116 & 116 & 121 & $80 *$ & 87 & 92 & 88 & 93 & 102 \\
\hline Proline & 316 & $365^{*}$ & 319 & $213 *$ & 267 & 251 & 199 & 243 & 213 \\
\hline
\end{tabular}

HP- higher protein diet, LP- lower protein diet, BF- breastfed

* Significant differences to BF $(\mathrm{p}<0.05)$. CHOP trial: P-values were obtained by a linear mixed model adjusted for study center and corrected for multiple testing. BeMIM trial: p-Values obtained by Kruskal-Wallis Tests, Karlsland Akeson et al.: p-values obtained by nonparametric tests of Kruskal-Wallis and Mann-Whitney and by analyses of variance, using the post-hoc test of Bonferroni/Dunn. 
Table 2 Mean and standard deviation $(\mu \mathrm{mol} / \mathrm{L})$ of plasma concentration of short-chain acylcarnitines (Carn) in high protein (HP) fed infants and low protein (LP) fed infants participating in the CHOP trial.

\begin{tabular}{lccc}
\hline Metabolite & LP & HP & $\boldsymbol{P}$ \\
\cline { 4 - 5 }$(\boldsymbol{\mu m o l} / \mathbf{L})$ & $(\mathbf{n}=\mathbf{2 6 0})$ & $(\mathbf{n = 2 6 2})$ & $\mathbf{L P ~ v s . ~ H P ~}$ \\
\hline Free Carnitine & $38(7.05)$ & $40(7.32)$ & $<0.0001$ \\
Carn C2 & $5.4(2.35)$ & $4.8(2.34)$ & 0.14 \\
Carn C3 & $313 \times 10^{-3}(0.1)$ & $479 \times 10^{-3}(0.2)$ & $<0.0001$ \\
Carn C4-OH & $72 \times 10^{-3}(0.03)$ & $67 \times 10^{-3}(0.05)$ & 1 \\
Carn C3-OH & $23 \times 10^{-3}(0.004)$ & $23 \times 10^{-3}(0.004)$ & 1 \\
Carn C3:1 & $6.7 \times 10^{-3}(0.002)$ & $6.5 \times 10^{-3}(0.002)$ & 1 \\
Carn C4 & $128 \times 10^{-3}(0.05)$ & $206 \times 10^{-3}(0.09)$ & $<0.0001$ \\
Carn C4:1 & $12 \times 10^{-3}(0.002)$ & $12 \times 10^{-3}(0.002)$ & 1 \\
Carn C5 & $95 \times 10^{-3}(0.04)$ & $154 \times 10^{-3}(0.06)$ & $<0.0001$ \\
Carn C5-M-DC & $41 \times 10^{-3}(0.006)$ & $39 \times 10^{-3}(0.006)$ & $<0.0001$ \\
Carn C5-OH & $39 \times 10^{-3}(0.009)$ & $45 \times 10^{-3}(0.01)$ & $<0.0001$ \\
Carn C5:1 & $18 \times 10^{-3}(0.007)$ & $21 \times 10^{-3}(0.008)$ & $<0.0001$ \\
Carn C5:1-DC & $19 \times 10^{-3}(0.009)$ & $18 \times 10^{-3}(0.01)$ & 1 \\
Carn C5-DC & $25 \times 10^{-3}(0.008)$ & $20 \times 10^{-3}(0.007)$ & $<0.0001$
\end{tabular}

P-values were obtained by a linear mixed model adjusted for study center and corrected for multiple testing. Adapted from Kirchberg et al. [12]

Table 3 Mean and standard deviation of ratios of the long-chain acylcarnitines C14, C16 and C18 to free carnitine in high protein (HP) fed infants and low protein (LP) fed infants participating in the CHOP trial.

\begin{tabular}{lccc}
\hline Ratio & LP & HP & P \\
\cline { 3 - 4 } & $\mathbf{( n = 2 6 0 )}$ & $\mathbf{( n = 2 6 2 )}$ & LP vs. HP \\
\hline Ratio C14 / free carnitine & $1.2 \times 10^{-3}(0.0003)$ & $1.0 \times 10^{-3}(0.0004)$ & $<0.0001$ \\
Ratio C16 / free carnitine & $2.6 \times 10^{-3}(0.0007)$ & $2.2 \times 10^{-3}(0.0008)$ & $<0.0001$ \\
Ratio C18 / free carnitine & $0.8 \times 10^{-3}(0.0002)$ & $0.7 \times 10^{-3}(0.0002)$ & $<0.0001$ \\
\hline
\end{tabular}

P-values were obtained from a linear mixed model adjusted for study center and corrected for multiple testing. Adapted from Kirchberg et al. [12] 


\section{Table headings}

\section{Table 1}

Plasma concentrations ( $\mu \mathrm{mol} / \mathrm{L}$ ) of amino acids in infants, who were formula fed or breastfed $(\mathrm{BF})$ in three different studies. HP- higher protein diet, LP- lower protein diet. BF- breastfed.

* Significant differences to BF $(<0.05)$. CHOP trial: P-values were obtained by a linear mixed model adjusted for study center and corrected for multiple testing. BeMIM trial: p-Values obtained by Kruskal-Wallis Tests, Karlsland Akeson et al.: p-values obtained by nonparametric tests of Kruskal-Wallis and Mann-Whitney and by analyses of variance, using the post-hoc test of Bonferroni/Dunn.

\section{Table 2}

Mean and standard deviation ( $\mu \mathrm{mol} / \mathrm{L})$ of plasma concentration of short-chain acylcarnitines (Carn) in high protein (HP) fed infants and low protein (LP) fed infants participating in the CHOP trial. P-values were obtained by a linear mixed model adjusted for study center and corrected for multiple testing. Adapted from Kirchberg et al. [12]

\section{Table 3}

Mean and standard deviation of ratios of the long-chain acylcarnitines C14, C16 and C18 to free carnitine in high protein (HP) fed infants and low protein (LP) fed infants participating in the CHOP trial. P-values were obtained from a linear mixed model adjusted for study center and corrected for multiple testing. Adapted from Kirchberg et al. [12] 
Figures

Figure 1

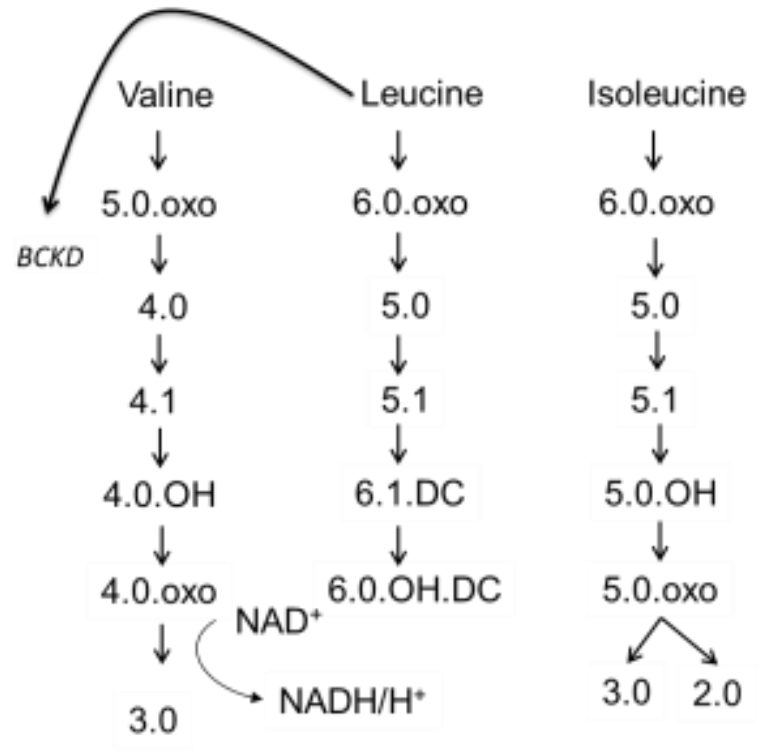

Figure 2
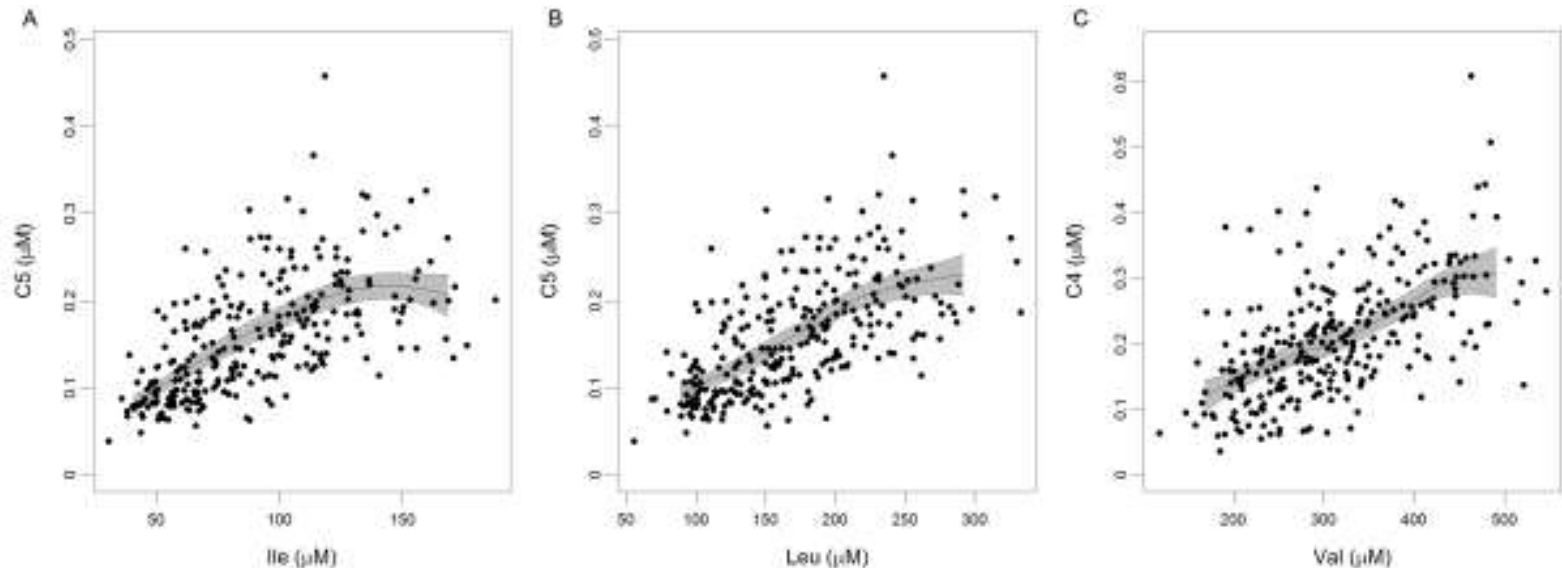


\section{Figure legends}

\section{Figure 1}

Degradation pathway of branched chain amino acids. Leucine activates the rate-limiting enzyme branch-chained keto-acid dehydrogenase (BCKD). The occurring short acyl-chains are bound to free carnitine.

\section{Figure 2}

The relation between branched chain amino acids (BCAA: Ile, Leu, Val) and their corresponding short-chain acylcarnitine indicates a concentration-dependent saturation of BCAA catabolism in infants. Modified from Kirchberg et al. [12] 